\title{
Design of a Portable Potentiostat with Dual-microprocessors for Electrochemical Biosensors
}

\author{
Chun-Yueh Huang \\ Department of Electrical Engineering, National University of Tainan, Taiwan
}

Copyright (C) 2015 by authors, all rights reserved. Authors agree that this article remains permanently open access under the terms of the Creative Commons Attribution License 4.0 International License

\begin{abstract}
In this paper, we design and implement a portable potentiostat by using dual-microprocessors for the signal processing of electrochemical biosensors. In our design approach, one of the microprocessors is used to design the programmable waveform generator, and the other microprocessor is used to measure the current of biosensors. The proposed potentiostat can perform general electrochemical analysis functions, including cyclic voltammetry, linear sweep voltammetry, differential pulse voltammetry, amperometry, and potentiometry. In the experiment, we adopt a commercial screen printed electrode immersed in potassium ferricyanide solution to test the performance of the proposed potentiostat and compare the proposed potentiostat's measured results with a commercial potentiostat's (CH Instrument Model: CHI1221) under the same test condition. The experimental results show that the proposed potentiostat has the merits of good accuracy, low cost, low power consumption, and high portability.
\end{abstract}

Keywords Potentiostat, Electrochemical Biosensors, Signal Processing Circuit

\section{Introduction}

Electrochemical biosensors, which can detect the particular target biomolecule to make a corresponding output change in the form of potential or current according to the biomolecule concentration, are commonly applied in DNA identification [1] and $\mathrm{pH}$ variation detection [2]. In electrochemical sensors, the potentiostat [3] is an important and indispensable device to maintain electrochemical stability.

Up to the present, two sorts of potentiostats have been frequently used: one is the commercial potentiostat designed for research in laboratory, and the other is the specific potentiostat developed for a particular sensor. The former is sophisticated, expensive, and non-portable, and the latter is simple, low-cost, and portable. In the light of low cost and portability, the latter seems to be more popular for researchers to study. In the past, many researchers devoted themselves to developing single-chip potentiostats for reducing chip's size and cost. Turner presented a basic CMOS integrated potentiostat [4], Kakerow presented a monolithic potentiostat [5], Bandyopadhyay proposed a multi-channel potentiostat designed in chip-type to reduce the size and the cost [6], and Huang proposed an integrated circuits (ICs) for monolithic implementation of a voltammetery potentiostat with a large dynamic current range ( $5 \mathrm{nA}$ to $1.2 \mathrm{~mA})$ and short conversion time $(10 \mathrm{~ms})$ [7]. In system realization, Huang proposed two portable potentiostats to mimic the commercial potentiostat's function to reduce the cost, using SOC based microprocessor and circuit components off the shelf to implement potentiostats on PCB [8-9].

In this paper, a portable potentiostat designed by using dual-microprocessors for the signal processing of electrochemical sensors is proposed to improve the accuracy of measurement. In Huang's designs [8-9], since a single microprocessor is used to generate waveform and measure the sensor's current in the meantime, a little timing mismatch will exist in waveform generator. In order to improve such a problem, we use two microprocessors to design the potentiostat. One is used to design the programmable waveform generator, and the other is used to measure the current of biosensors. The two microprocessors are communicated with one memory card to obtain experimental parameters and to work independently. This design approach can avoid the mutual interference between current measurement and waveform generation to improve measurement accuracy. The proposed portable potentiostat can be used to perform the electrochemical measurements of biosensors outside the laboratory.

\section{The Proposed Potentiostat}

\subsection{Principle of a Potentiostat's Operation}

An electrochemical biosensor is composed of a working electrode (W), a reference electrode (R), and a counter 
electrode (C). The working electrode with the biomolecular probes attached can generate the reaction with the target. In an electrochemical biosensor, a potentiostat is an electronic device used to maintain electrochemical stability and to convert the biosensor's output into an analog signal [10]. Fig. 1(a) is a potentiostat's operation diagram, which can be implemented by the electronic components as shown in Fig. 1(b). The operational amplifier on the right establishes the control loop to accomplish the potentiostatic control while the integrator on the left converts the current flowing through the $\mathrm{W}$ to a voltage for digitization and readout.

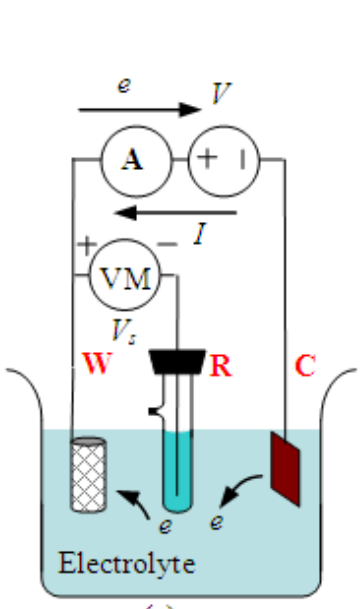

(a)

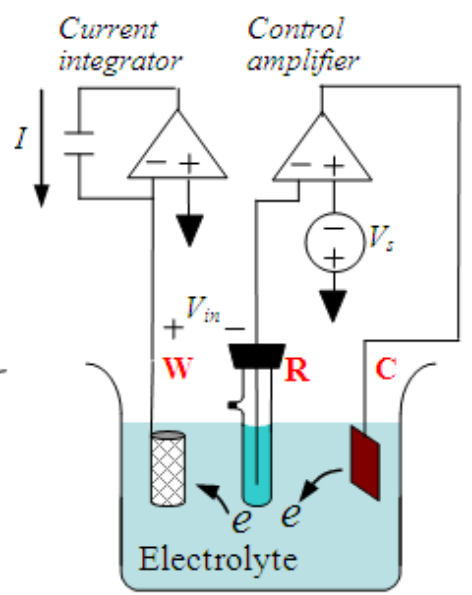

(b)
Figure 1. A potentiostat's operation: (a) Concept diagram, (b) Implementation by using electronic components.

\subsection{Design of a Portable Potentiostat}

Fig. 2 shows the circuit diagram of a portable potentiostat designed with dual-microprocessors. The proposed potentiostat is mainly constructed by two mixed-signal microprocessors (MCU1, and MCU2), a voltage amplifier, an auto-range current-to-voltage converter, a voltage follower, an operational amplifier, a DC-DC power converter, and an SD memory card. The microprocessor MCU2 is used to communicate with PC through USB interface and to get the experimental parameters of the potentiostat from PC, and these parameters will be stored in an SD memory card. The other microprocessor MCU1 is used to generate the required signal waveform by 12-bit digital to analog converter according to the experimental parameters. The signal in range of $0 \sim 2.4 \mathrm{~V}$ will be amplified to $0 \sim 3.2 \mathrm{~V}$ by a voltage amplifier to extend the scan range of the potentiostat. The two microprocessors are communicated with the interface of memory card to obtain the desired experimental parameters. After the experimental parameters are set up, two microprocessors can be operated independently. MCU1 is in charge of signal waveform generation, and MCU2 is in charge of biosensor's current measurement. During the process of experiment, the potentiostat is controlled by the microcontrollers. The power supply can be provided by a USB charger, without using AC power supply. This design approach can increase the portability and the ability of standalone operation.

In the proposed potentiostat, an auto-range current to voltage converter, which contains a transimpedance amplifier and a set of resistors $(10 \mathrm{M} \Omega, 1 \mathrm{M} \Omega, 100 \mathrm{k} \Omega, 10$ $\mathrm{k} \Omega, 1 \mathrm{k} \Omega$, and $100 \Omega$ ), is used to measure the current flowing through the solution between the working and the counter electrodes. It can be used to automatically convert the sensor's current range from $\pm 16 \mathrm{~mA}$ to $\pm 160 \mathrm{nA}$ by the microprocessor to select the suitable resistor.

The C8051F005 chip is an 8051 based CPU with 12-bit digital-to-analog converters (DACs), 12-bit analog-to-digital converters (ADCs), and digital peripherals. According to the specification of C8051F005 chip, the output range of DAC is between 0 and $2.4 \mathrm{~V}$, and hence $\mathrm{DAC}$ can generate positive potential only and can't generate negative potential. In order to realize the function of cyclic voltammetry, the potentiostat needs to generate a sweep potential in both forward and reverse directions. Thus, two 12bit DACs (DAC1 and DAC0) are used to generate a scan potential between 0 and $2.4 \mathrm{~V}$, and a $1.6 \mathrm{~V}$ offset potential, respectively. The potential of the working electrode $\left(\mathrm{V}_{\mathrm{W}}\right)$ is set as $1.6 \mathrm{~V}$ by the voltage on the operational amplifier's virtual "ground" node. Based on the preset scan potential, the microprocessor generates a triangular-type signal, and then the DAC1 will transfer it into a triangular-type voltage between 0 and $2.4 \mathrm{~V}$, which will be amplified to 0 and 3.2 $\mathrm{V}$, and be acted as the setting voltage $\left(\mathrm{V}_{\mathrm{set}}\right)$. In the operational amplifier, a negative feedback loop controls the potential of the reference electrode $\left(V_{R}\right)$ to be equal to the setting voltage $\left(\mathrm{V}_{\text {set }}\right)$. According to this approach, the scan voltage $\mathrm{V}_{\mathrm{WR}}$ will be obtained in the range of $-1.6 \mathrm{~V}$ to 1.6 $\mathrm{V}$.

The design specifications of the proposed cyclic voltammetry potentiostat are described as follows: the range of the programmable scan potentials $\left(\mathrm{V}_{\mathrm{WR}}\right)$ is between -1.6 $\mathrm{V}$ and $1.6 \mathrm{~V}$ under the resolution $1 \mathrm{mV}$, the minimum programmable scan rate is $5 \mathrm{mV} / \mathrm{s}$, and the auto-range of the measured current $\left(\mathrm{I}_{\mathrm{WC}}\right)$ is from $\pm 16 \mathrm{~mA}$ in the resolution of $8 \mu \mathrm{A}$ to $\pm 160 \mathrm{nA}$ in the resolution of $80 \mathrm{pA}$.

\subsection{Auto-range Current-to-voltage Converter}

In the potentiostat, the auto-range current to voltage converter is used to measure the biosensor's current. In the auto-range current to voltage converter, the resistor's selection strategy is based on a sequential search algorithm to find a suitable resistor for current-to-voltage conversion. At first the $100 \Omega$ resistor will be selected, and then the converted voltage will be measured by ch-4 ADC of MCU2. The measured voltage will be checked whether it is in the range of (1).

$$
1.6-0.15<V_{c h 4}<1.6+0.15
$$




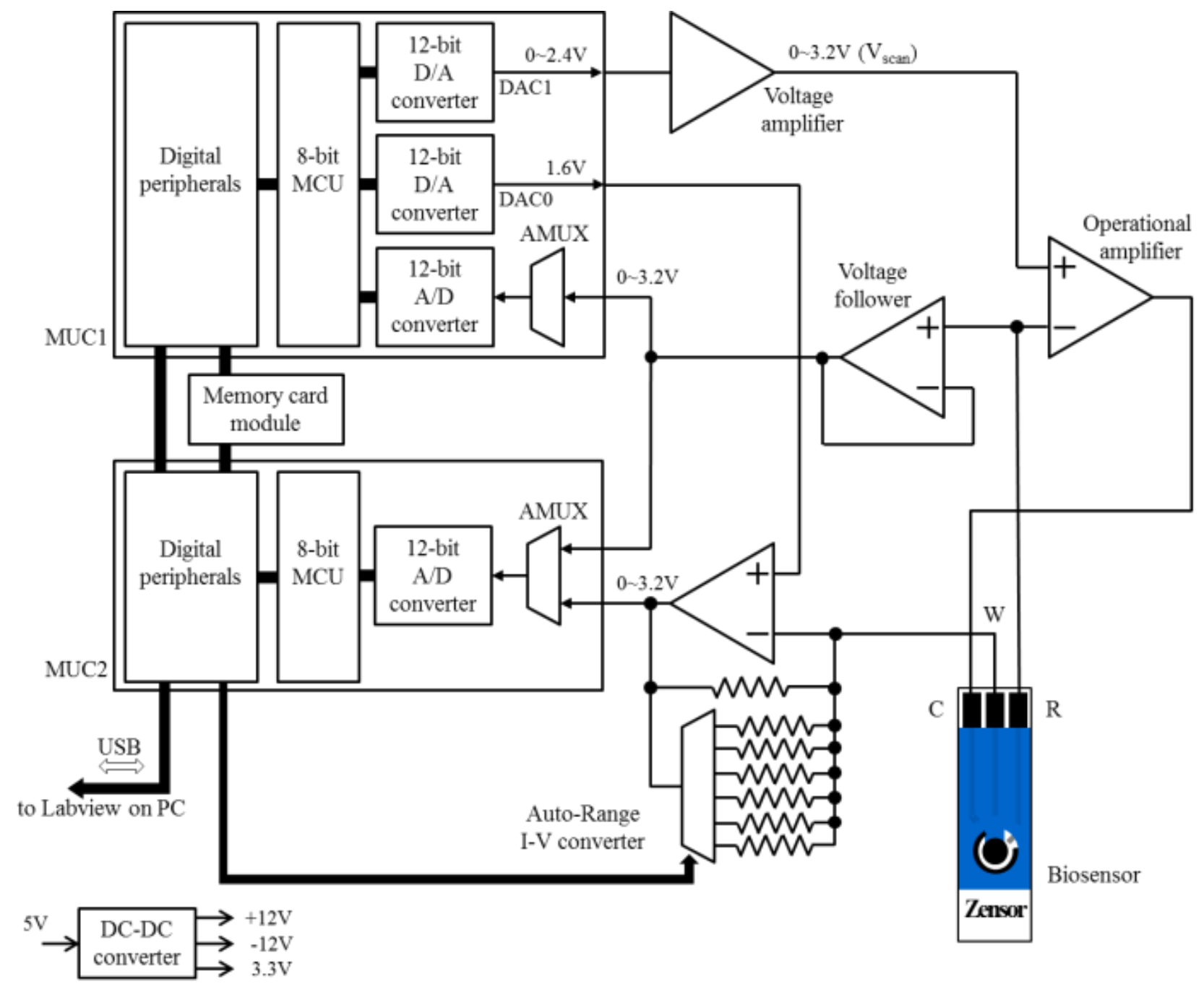

Figure 2. The circuit diagram of a portable potentiostat designed with dual-microprocessors.

If the range of the converted voltage is in the condition of (1), which means that the presented resistance is too small to be used, then the resistance will be enlarged ten times. The foregoing procedure will be continued until the range of the converted voltage satisfies the condition of (2) or until the resistance reaches $10 \mathrm{M} \Omega$.

$$
V_{c h 4}<1.6-0.15 \text { or } V_{c h 4}>1.6+0.15
$$

After the suitable resistance is found, the resistance will be recorded and sent to the next cycle current-to-voltage conversion. In the new converting cycle, according to the variation of the biosensor's current, the previous converting resistor may not suit the present current conversion. So, the converting resistor will be changed to a larger resistance if the condition of (1) is satisfied. Otherwise, the converting resistor will be changed to a smaller resistance if the condition of (3) is satisfied.

$$
V_{c h 4}>3.05 \text { or } V_{c h 4}<0.15
$$

\subsection{Personal User Interface}

The experiment is controlled by a PC running LabVIEW software, via a USB interface to communicate with the microprocessor. The personal user interface is shown in Fig. 3 , which contains cyclic voltammetry, linear sweep voltammetry, differential pulse voltammetry, amperometry, and potentiometry. In the cyclic voltammetry mode, we can see the experimental parameter setting table, the real-time cell potential, the real-time sensor's current, and voltammogram diagram. Before the experiment, the user has to set up some experimental parameters, such as the scan range which can be set by the "initial" and "final" settings, the scan rate, and the scan cycles. The scan rate is determined by the number of increments in the output voltage (called "steps") and the number of seconds fixed in each step. 


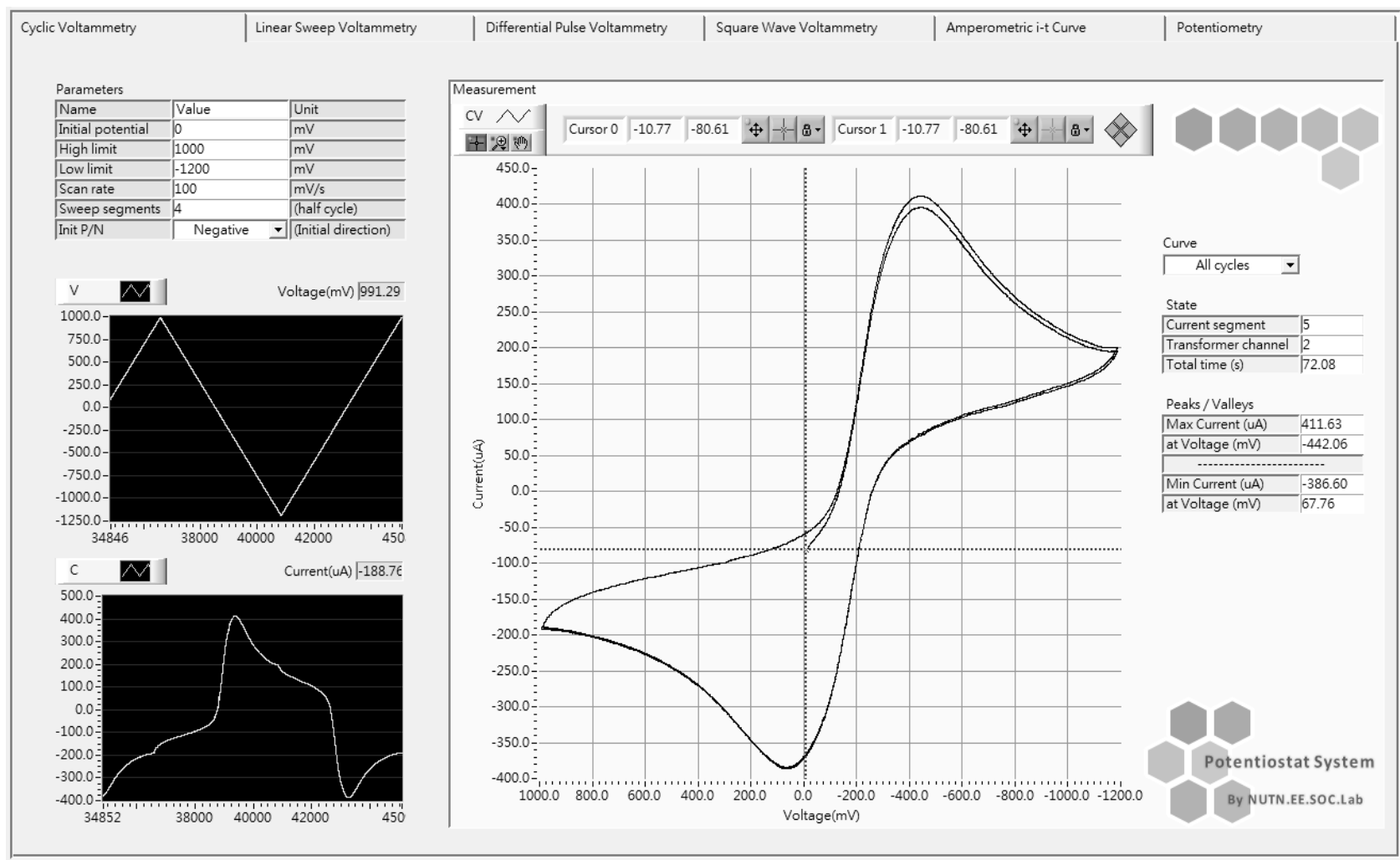

Figure 3. The Labview operation interface of PC.

During the experiment, the LabVIEW program controls the experimental procedure and collects the measured data from the potentiostat. Before the LabVIEW program plots the cyclic voltammogram, a digital 'weighted averaging filter' [11] is used to smooth the measured current signal. The algorithm of weighted averaging filter is

$$
Y(t)=\frac{[(L-1) \times Y(t-1)]+X(t)}{L}
$$

where $X(t)$ is the value of new input sample, $Y(t-1)$ is the value of previous output, $Y(t)$ is the value of present output, and $L$ is the weighted factor of the filter.

\subsection{SD Memory Card}

The SD memory card is used to store the experimental parameters and the measured results of the potentiostat. In SD memory card system, there are two alternative communication protocols: SD and SPI. In order to communicate the memory card with the microcontroller, the SPI communication protocol, which is one of the built-in communication protocols of $\mathrm{C} 8051 \mathrm{~F} 005$, is used to reduce the design-in effort to minimum. Through the signals of CS, CLK, DataIn, and DataOut, we can connect the SPI interface of C8051F005 with the SD memory card. All communications between the microcontroller and the memory card are controlled by the microcontroller. The FAT16 format is adopted to store data in SD card. The measured data of each experiment will be stored in one text file and can be retrieved to analyze and to plot the experimental diagram by PC.

\subsection{Experimental Results}

In the experiment, we adopt a commercial screen printed electrode as a blank biosensor to be immersed in potassium ferricyanide solution to test the performance of the proposed potentiostat and compare the measured results with a commercial potentiostat's (CHI1221) under the same test condition. In other words, the same biosensor and ferricyanide solution will be tested by the proposed potentiostat and the commercial potentiostat alternatively. Fig. 5 shows a comparison diagram of the cyclic voltammetry measurements performed by the proposed potentiostat and a commercial potentiostat. The scanned voltage is between $-1 \mathrm{~V}$ and $+1 \mathrm{~V}$ at a scan rate of $100 \mathrm{mV} / \mathrm{s}$.

Fig. 6 (a) and (b) are the cyclic voltammograms of the blank biosensor, which are immersed in the standard test solution with different concentration measured by the proposed potentiostat, and the commercial one. The cyclic voltammetry measurements for the blank biosensor are respectively performed in $1 \mathrm{mM}, 3 \mathrm{mM}, 5 \mathrm{mM}$ potassium ferricyanide solution, at a scan rate of $100 \mathrm{mV} / \mathrm{s}$, and the scanned voltage is between $-0.6 \mathrm{~V}$ and $+0.8 \mathrm{~V}$. Fig. 7 exhibits the relationship between the target concentration and anodic peak currents, which is re-plotted in accordance with Fig. 6. The current response for the blank biosensor is monotonically increased from 8 to $31 \mu \mathrm{A}$, when the concentration of potassium ferricyanide solution is increased from $1 \mathrm{mM}$ to $5 \mathrm{mM}$. The measured results of the proposed potentiostat are almost the same as the commercial potentiostat's. 


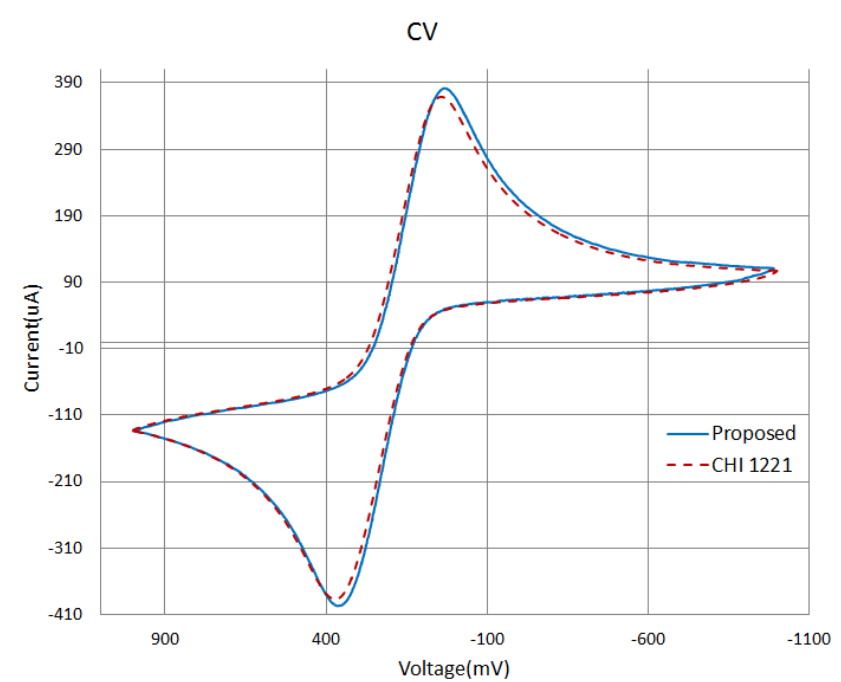

Figure 5. A comparison diagram of the cyclic voltammetry measurements performed by the proposed potentiostat and a commercial potentiostat (CHI1221).

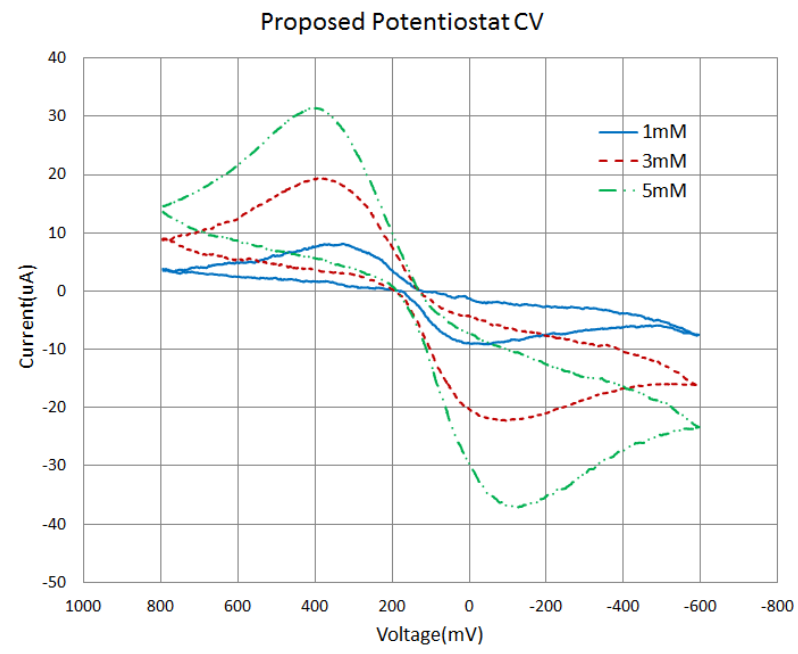

(a)

$\mathrm{CHI} 1221 \mathrm{CV}$

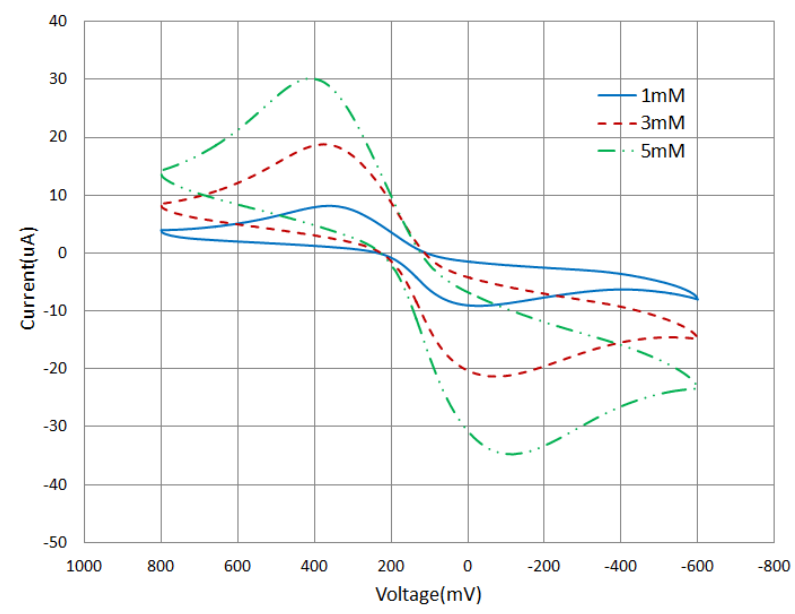

(b)

Figure 6. The cyclic voltammograms of the blank biosensor which are immersed in the standard test solution with different concentration measured by (a) the proposed potentiostat, and (b) the commercial potentiostat.

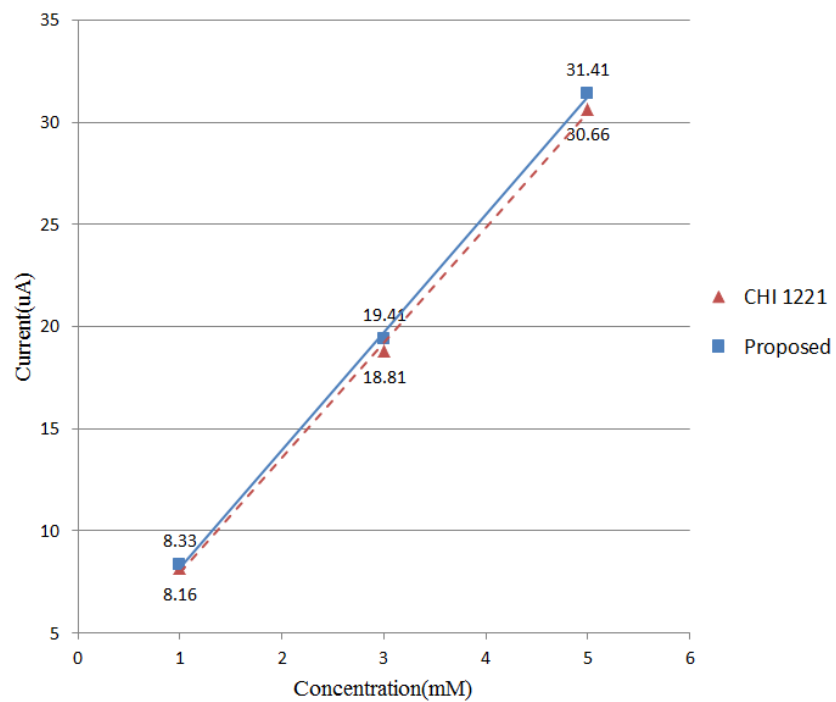

Figure 7. The relationship between the target concentration and the anodic peak current.

In addition to the demonstration of the function of cyclic voltammetry, Fig. 8 shows the comparison results of differential pulse voltammetry, where the scan voltage is from $-0.4 \mathrm{~V}$ to $1 \mathrm{~V}$, the pulse amplitude, pulse width, and pulse period are $50 \mathrm{mV}, 50 \mathrm{~ms}$, and $200 \mathrm{~ms}$, respectively. The increased potential of each pulse is $5 \mathrm{mV}$, and the sampling time is $17 \mathrm{~ms}$. According to the experimental results, we can observe that the proposed potentiostat's performance can achieve the accuracy as good as the commercial one's.

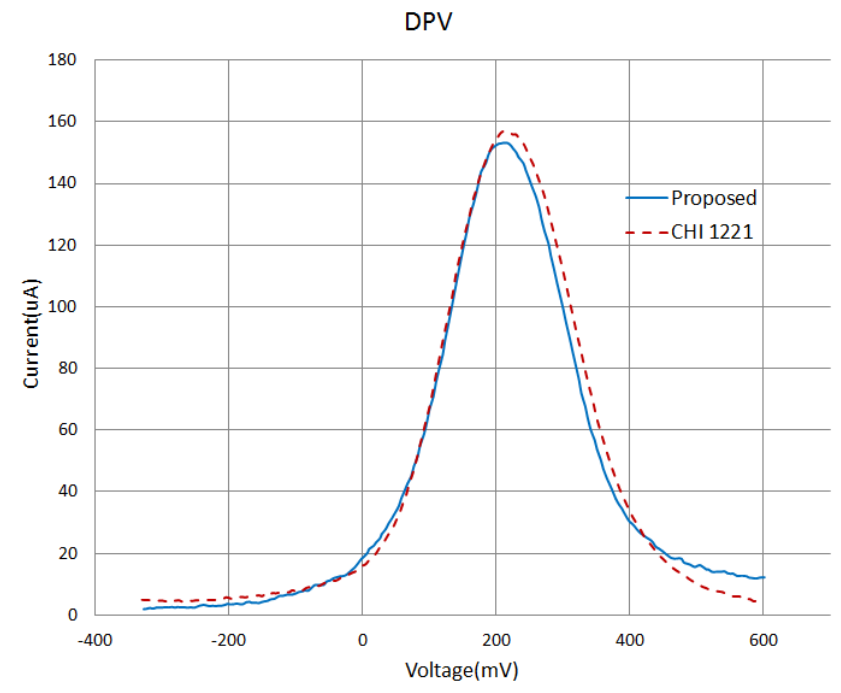

Figure 8. A comparison diagram of the differential pulse voltammetry measurements performed by the proposed potentiostat and a commercial potentiostat (CHI1221).

\section{Conclusions}

In this paper, we propose a portable potentiostat designed by dual-microprocessors for the signal processing of electrochemical biosensors. The proposed potentiostat can 
perform general electrochemical analyses, such as cyclic voltammetry, linear sweep voltammetry, differential pulse voltammetry, amperometry, and potentiometry. Comparing the proposed potentiostat's performance with the commercial potentiostat's, the proposed potentiostat's performance is as good as the commercial one's according to the experimental results. In addition, the proposed potentiostat has the merits of low cost, low power consumption, and high portability. In the future, the proposed potentiostat can be cooperated with real biosensors to be applied in home-care system.

\section{Acknowledgments}

This work is supported by MOE 104-2221-E-024 -017.

\section{REFERENCES}

[1] I. J. Chen, I. M. White, "Sandwich structure electrochemical assay for single stranded DNA detection," IEEE Sensors, pp. 470-473 (2010).

[2] F. Shahrokhi, K. Abdelhalim, D. Serletis, P. L. Carlen, R. Genov, "The 128-Channel Fully Differential Digital Integrated Neural Recording and Stimulation Interface," IEEE Trans. on Biomedical Circuits and Systems, Vol. 4 , pp. $149-161$ (2010).

[3] H. Glaster, in pH Measurements-Fundamentals, Methods, Applications, Instruments, Wiley-VCH, New York. (1991).

[4] R. F. B. Turner, D. J. Harrison, and H. P. Baltes, "A CMOS potentiostat for amperometric chemical sensors," IEEE $J$. Solid-State Circuits, vol. 22, pp. 473-478, (1987).

[5] R. G. Kakerow, H. Kappert, E. Spiegel, and Y. Manoli, "Low power single chip CMOS potentiostat," in Proc. Eur. Conf. Solid State Transducers, pp. 142-145, (1995).

[6] A. Bandyopadhyay, G. Mulliken, G. Cauwenberghs, and N. Thakor, "VLSI potentiostat array for distributed electrochemical neural recording," in Proc. IEEE Int. Sym. Circuit and Syst., pp. 740-743, (2002).

[7] C.Y. Huang, I.J. Chao, J.L. Thomas, H.W. Wei, Y.F. Liang, B.D. Liu, M.H. Lee, and H.Y. Lin, "Integrated potentiostat for electrochemical sensing of urinary 3-hydroxyanthranilic acid with molecularly imprinted poly(ethylene-co-vinyl alcohol)," Biosensors and Bioelectronics, In Press, Available online 19 August 2014.

[8] C. Y. Huang, T. C. Tsai, J. L. Thomas, M. H. Lee, B. D. Liu and H. Y. Lin," Urinalysis with molecularly imprinted poly(ethylene-co-vinyl alcohol) potentiostat sensors," Biosensors and Bioelectronics, Vol. 24, Issue 8, p.2611-2617, (2009).

[9] C. Y. Huang, M. J. Syu, C. H. Chang, Y. S. Chang, B. D. Liu, and T. C. Chou, "A portable potentiostat for the bilirubin-specific sensor prepared from molecular imprinting," Biosensors and Bioelectronics, Vol. 22, Issue 8, pp. 1694-1699, (2007).

[10] P. M. Levine, P. Gong, R. Levicky, and K. L. Shepard, "Active CMOS Sensor Array for Electrochemical Biomolecular Detection," IEEE J. Solid-State Circuits, vol. 43, pp. 1859-1871 (2008).

[11] M. K. Ozkan, M. I. Sezan, and A. M. Tekalp, "Adaptive motion-compensated filtering of noisy image sequences," IEEE Trans. Circuits Syst. Video Technol., vol. 3, pp. 277-290, (1993). 